\title{
Depth dependence of optical property beyond the critical thickness of an InGaN film
}

\author{
Chih-Chung Teng ${ }^{\mathrm{a}}$, Hsiang-Chen Wang ${ }^{\mathrm{b}}$, Tsung-Yi Tang ${ }^{\mathrm{b}}$, Yen-Cheng Lu ${ }^{\mathrm{b}}$, \\ Yung-Chen Cheng ${ }^{\mathrm{b}}$, C.C. Yang ${ }^{\mathrm{a}, \mathrm{b}, \mathrm{c}, *}$, Kung-Jen Ma ${ }^{\mathrm{d}}$, Wei-Ming Wang, \\ Chi-Wei Hsu ${ }^{\mathrm{e}}$, L.C. Chen ${ }^{\mathrm{e}}$ \\ ${ }^{a}$ Graduate Institute of Electronics Engineering, National Taiwan University, 1, Roosevelt Road, Sec. 4, Taipei, Taiwan, ROC \\ ${ }^{\mathrm{b}}$ Graduate Institute of Electro-Optical Engineering, National Taiwan University, 1, Roosevelt Road, Sec. 4, Taipei, Taiwan, ROC \\ ${ }^{\mathrm{c}}$ Department of Electrical Engineering, National Taiwan University, 1, Roosevelt Road, Sec. 4, Taipei, Taiwan, ROC \\ ${ }^{\mathrm{d}}$ Department of Mechanical Engineering, Chung Hua University, Hsinchu, Taiwan, ROC \\ ${ }^{\mathrm{e}}$ Center for Condensed Matter Sciences, National Taiwan University, Taipei, Taiwan, ROC
}

Available online 18 January 2006

\begin{abstract}
We study the depth-dependent variation of optical property beyond the critical thickness in an InGaN thin film. In the sample, both free-carrier and localized-state recombination activities are observed. The emission peak corresponding to the localized states in photoluminescence (PL) measurement becomes more prominent with increasing sample depth, implying stronger clustering in the deeper layers. Although the PL spectral peak variation is weak, that of cathodoluminescence (CL), corresponding to the activities of the localized states, shows a clear red shift trend with depth. The red shift trend is attributed to the stronger clustering behavior and possibly stronger quantum-confined Stark effect in the nano-clusters, which is due to the residual strain beyond the critical thickness, in a deeper layer.
\end{abstract} (C) 2005 Elsevier B.V. All rights reserved.

PACS: 68.37.-d; 78.45. $+\mathrm{h} ;$ 78.55.Cr: 78.60.Hk

Keywords: A1. Segregation; B1. Nitride; B3. Light-emitting diodes

\section{Introduction}

The critical thickness of InGaN grown on GaN, beyond which the strain built at the hetero-structure interface is well relaxed, has been widely studied [1-3]. It was calibrated that for average indium contents between 5 and $20 \%$, the critical thickness ranges from 130 to $60 \mathrm{~nm}$ [1]. However, because of the large lattice mismatch between $\mathrm{GaN}$ and $\mathrm{InN}$, their low miscibility usually leads to nonuniform structures of InGaN compounds through spinodal decomposition in the region beyond the critical thickness. To release strain energy, indium aggregation and phase separation can usually result in nano-scale indium-rich

\footnotetext{
${ }^{*}$ Corresponding author. Graduate Institute of Electro-Optical Engineering, National Taiwan University, 1, Roosevelt Road, Sec. 4, Taipei, Taiwan, ROC. Tel.: + 886223657624 ; fax: + 886223652637.

E-mail address: ccy@cc.ee.ntu.edu.tw (C.C. Yang).
}

clusters and compositional fluctuations [4-6]. In particular, when a perturbation is introduced, such as thermal annealing, spinodal decomposition will enhance the clustering process $[7,8]$. Besides the local aggregation phenomenon, a more global behavior, called indium pulling [9-11], was also discovered. Indium pulling means that in either a quantum well or a hetero-structure of InGaN, indium composition increases with growth thickness. This behavior is usually attributed to the weaker incorporation of indium near the interface of InGaN growth that is again due to the lattice mismatch between $\mathrm{GaN}$ and nominal InGaN. As the growth thickness increases, indium incorporation becomes stronger and hence the indium composition becomes higher. However, it is usually believed that indium pulling occurs only in the strained region near the interface, i.e., within the critical thickness. Besides the indium pulling behavior, in a relatively thicker InGaN film, it was also speculated that a deeper layer 
might have experienced the thermal annealing process during the growth of a shallow layer [12]. This process would change the clustering structures and lead to different photon emission properties.

In this paper, we report the depth-dependent variations of optical property in an InGaN thin film grown on a $\mathrm{GaN}$ buffer layer. Photoluminescence (PL) and cathodoluminescence $(\mathrm{CL})$ measurements are used for demonstrating the trends of photon emission spectral variations among different depths. This paper is organized as follows: In Section 2, the sample structures are described. Then, we present the depth-dependent PL and CL results in Section 3. Finally, discussion and conclusions are made in Section 4.

\section{Sample structures}

The InGaN thin film sample was grown on $c$-plane sapphire with MOCVD. After the 800-nm GaN buffer layer, an 800-nm un-doped InGaN thin film was grown at $800{ }^{\circ} \mathrm{C}$. The nominal indium content of the InGaN layer is about $20 \%$. The critical thickness of the sample is estimated to be around $60 \mathrm{~nm}$ [1]. Therefore, the major part of the InGaN film is expected to be free from the hetero-structure-induced strain. Because PL measurement can monitor the optical properties only in a shallow layer under such a high absorption condition, the as-grown sample was etched with various depths for depth-dependent optical study. Four samples were prepared with the dry etching depths of 0 (un-etched), 150, 300, and $450 \mathrm{~nm}$.

Fig. 1 shows the scanning electron microscopy (SEM) images on the surfaces of the un-etched sample (a) and the sample of 300-nm etching depth (b). The pattern in either image looks similar to that of a material with strong spinodal decomposition [13]. The scale sizes of these patterns are in the range of $400-600 \mathrm{~nm}$. Fig. 2 shows a typical high-resolution transmission electron microscopy (HRTEM) image. In this image, indium-rich clusters, as indicated with thick arrows, can be clearly seen. The HRTEM investigation was performed using a Philips Tecnai F30 field-emission electron microscope using an accelerating voltage of $300 \mathrm{kV}$ and a probe forming lens of $C_{\mathrm{s}}=1.2 \mathrm{~mm}$. The current density focused onto the samples was estimated to be lower than $16 \mathrm{~A} / \mathrm{cm}^{2}$. The clusters in Fig. 2 form potential minima and hence localized states to trap carriers for effective radiative recombination.

\section{Measurement results}

Fig. 3 shows the PL spectra of various etching depths at $10 \mathrm{~K}$. Two spectral peaks around 2.7 and $3.05 \mathrm{eV}$ can be clearly seen in each spectrum. With 0 and $150 \mathrm{~nm}$ etching depths, the high-energy peak dominates; however, with deeper etching, the low-energy peak becomes stronger. The tails around $2.3 \mathrm{eV}$ represent the defect-induced luminescence [14]. The inset of Fig. 3 shows the counterparts at $300 \mathrm{~K}$. Here, only the low-energy peaks around $2.7 \mathrm{eV}$ are
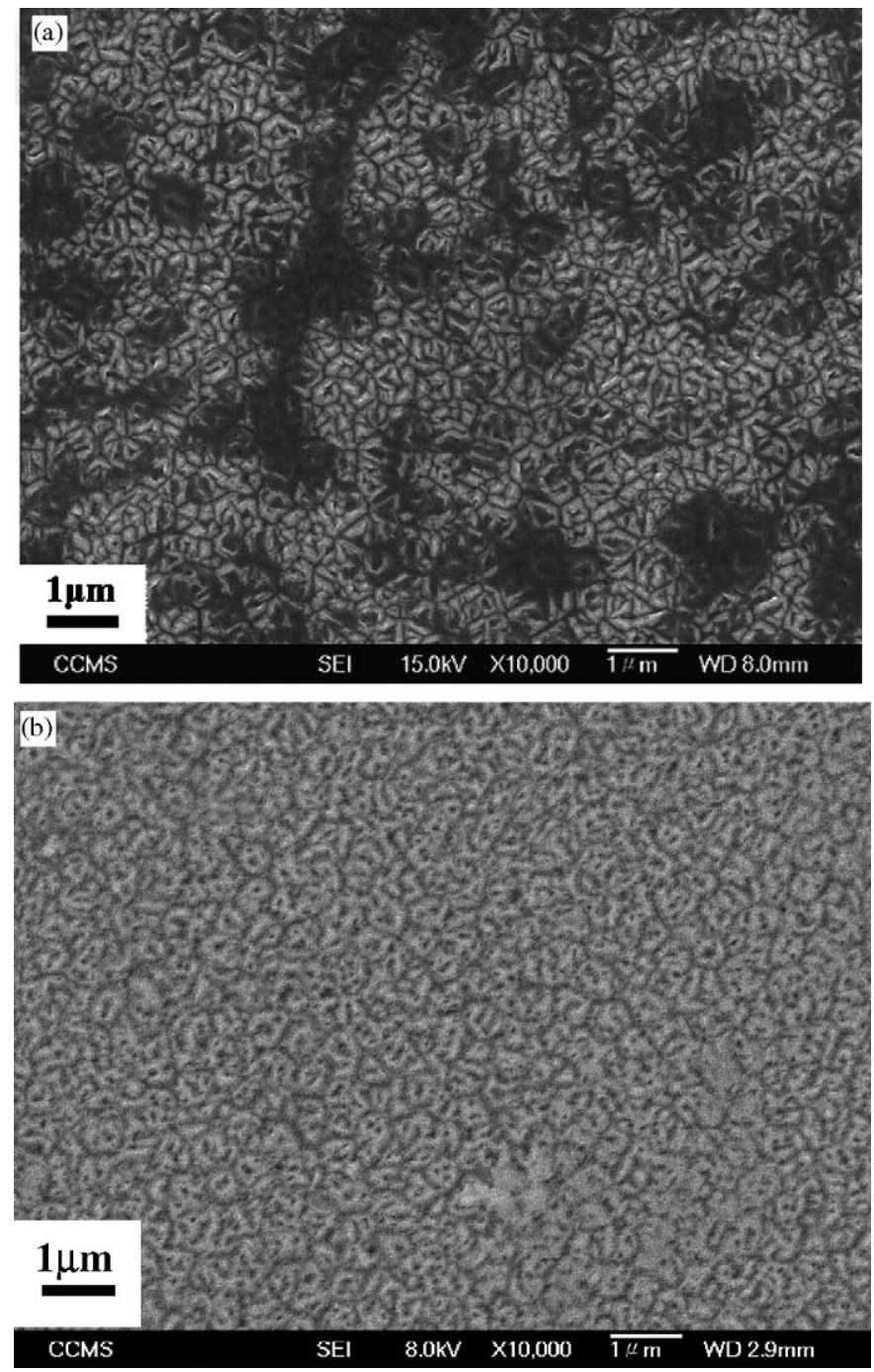

Fig. 1. SEM images on the surfaces of the un-etched sample (a) and the sample of 300-nm etching depth (b).

observed for all the samples of different etching depths. The defect-induced luminescence becomes stronger at room temperature. Its intensity increases with increasing etching depth. Because indium aggregation is expected in such an InGaN compound, the low-energy PL peak is supposed to originate from the localized carriers in the formed indium-rich clusters. The high-energy PL peak results from the carriers in the energy states of the background InGaN compound, on which clusters distribute.

Fig. 4 shows the variations of PL spectral peak positions with temperature of various etching depths. The lowenergy peaks illustrate the typical S-shape behavior of localized states. Because the low-energy peak in the unetched sample is unclear, only the high-energy peak is shown in Fig. 4 for this sample. In all the samples, the highenergy peaks red shift with temperature. The variations are steeper than the band gap shrinkage trend of phonon effect that can possibly be due to carrier relaxation into lower 


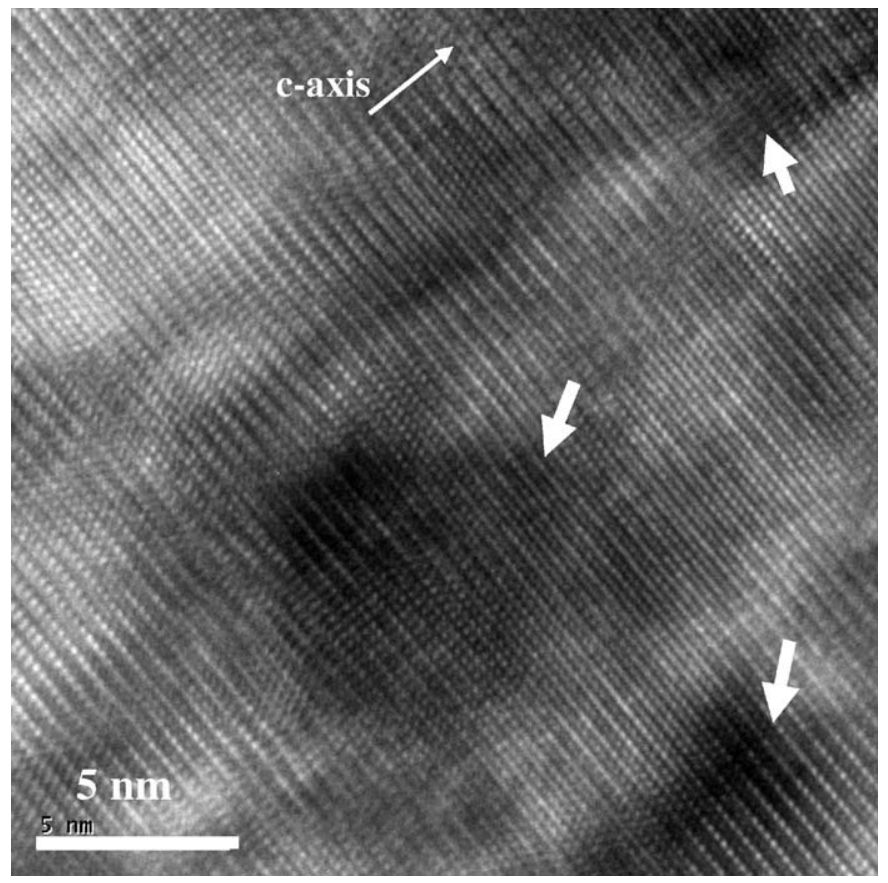

Fig. 2. A typical HRTEM image showing the indium-rich clusters.

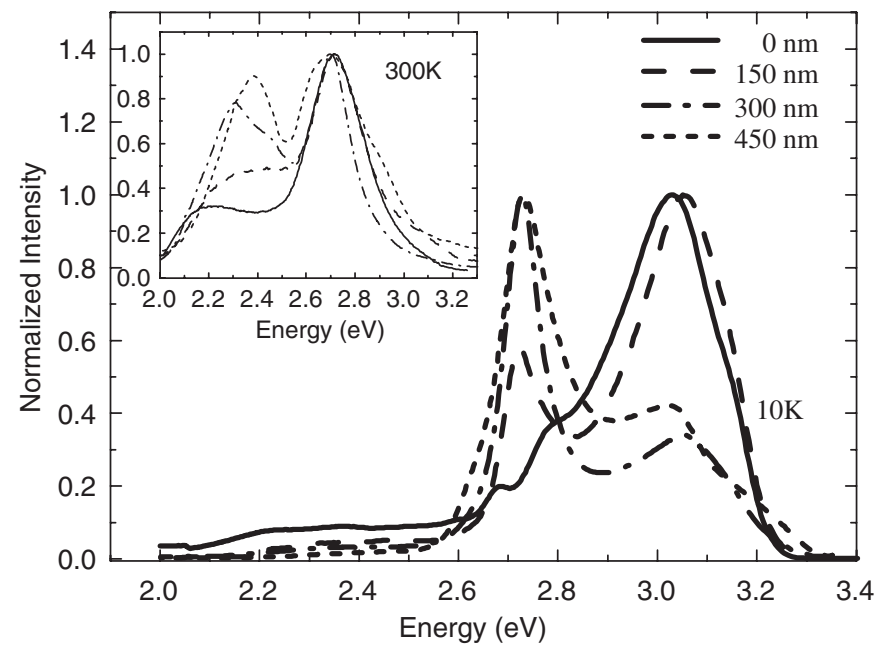

Fig. 3. Normalized PL spectra of different etching depths at $10 \mathrm{~K}$. The inset shows the counterparts at $300 \mathrm{~K}$.

energy states. They tend to merge into the low-energy peaks at room temperature. The merge of the high- and low-energy PL peaks also indicates the carrier relaxation process. The unclear low-energy peak in the un-etched sample reveals that clustering is relatively weaker in the shallow layer. Basically, the variation of PL peak at various depths is weak (in the range of $30 \mathrm{meV}$ ). The high-energy peaks of the deeper layers are slightly blue-shifted from those of the surface. However, no clear trend exists for the low-energy peak.

Fig. 5 shows the variations of integrated PL intensity versus temperature at various depths. One can see that the radiative efficiency decreases with increasing depth. Be-

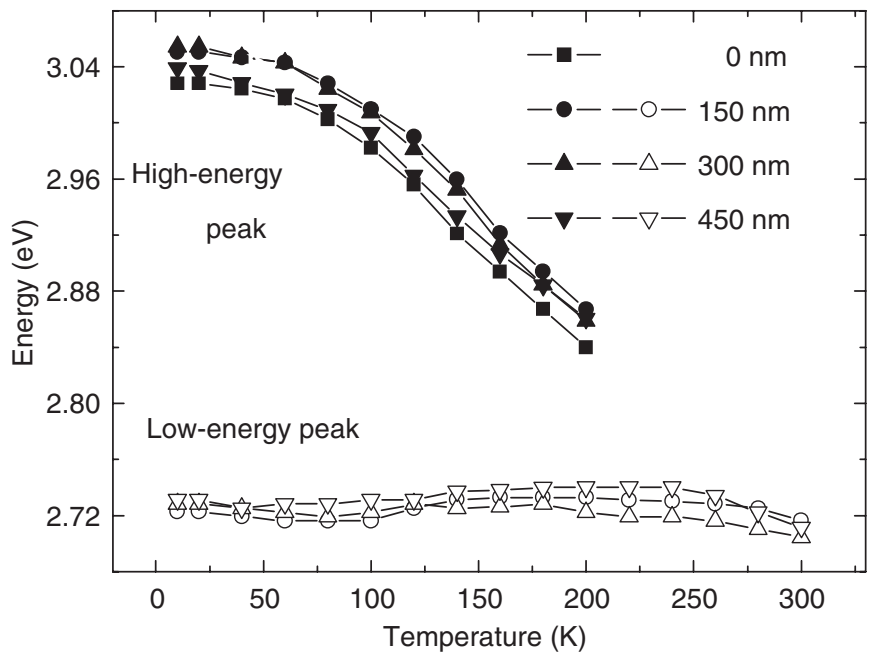

Fig. 4. PL spectral peak positions versus temperature of different etching depths.

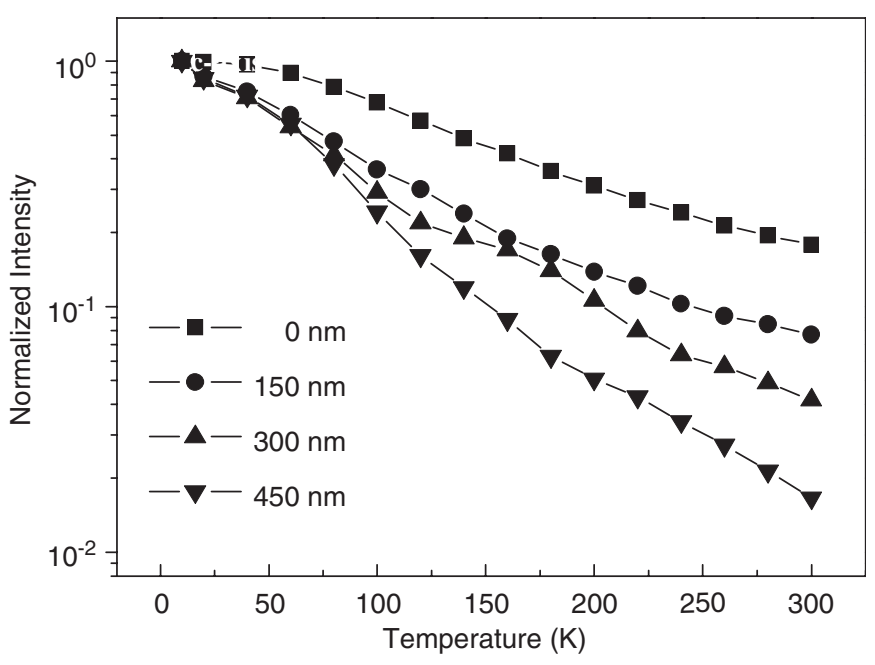

Fig. 5. Normalized integrated PL intensities versus temperature of different etching depths.

cause it was speculated from Fig. 3 that clustering or carrier localization (for effective radiative recombination) was stronger in a deeper layer, the lower radiative efficiency indicated in Fig. 5 implies a higher defect density in a deeper layer. This implication is consistent with the variation of the feature between 2 and $2.5 \mathrm{eV}$ in Fig. 3. Note that the process of dry etching might create damages and defects on the sample surfaces. Such a process can enhance the density of non-radiative recombination centers, leading to the results in Figs. 3 and 5. However, the CL spectra in Fig. 6 also show the increasing defectinduced-luminescence intensity with depth. Such results imply that the defect density does increase with depth in the as-grown sample.

Fig. 6 shows the CL spectra of various etching depths at $300 \mathrm{~K}$ with a fixed electron energy at $2 \mathrm{keV}$. The defectinduced luminescence between 2 and $2.6 \mathrm{eV}$ is generally stronger in a deeper layer. The peak of the localized-state- 
induced luminescence around $2.8 \mathrm{eV}$ red-shifts with increasing depth. Fig. 7 shows the CL spectra of various electron energies measured from the surface of the unetched sample at $300 \mathrm{~K}$. They show the similar trend to that of Fig. 6. The major peak red-shifts with increasing electron penetration depth. The defect-induced luminescence intensity also increases with the penetration depth, indicating that the defect density is higher in a deeper layer, as mentioned before. Fig. 8 summarizes the CL spectral peak variations of the two measurements leading to Figs. 6 and 7. Those of the measurement for Fig. 6, but with $8 \mathrm{keV}$ in electron energy, are also shown in Fig. 8 for comparison. Here, the trends of varying etching depth and varying electron energy are the same. Because the increase of electron energy also implies the increase of probing depth, Fig. 8 shows the decreasing CL spectral peak energy with depth in the sample under study. Note that the penetration depths of electron beam with energies of 3, 5, 8, and $15 \mathrm{keV}$

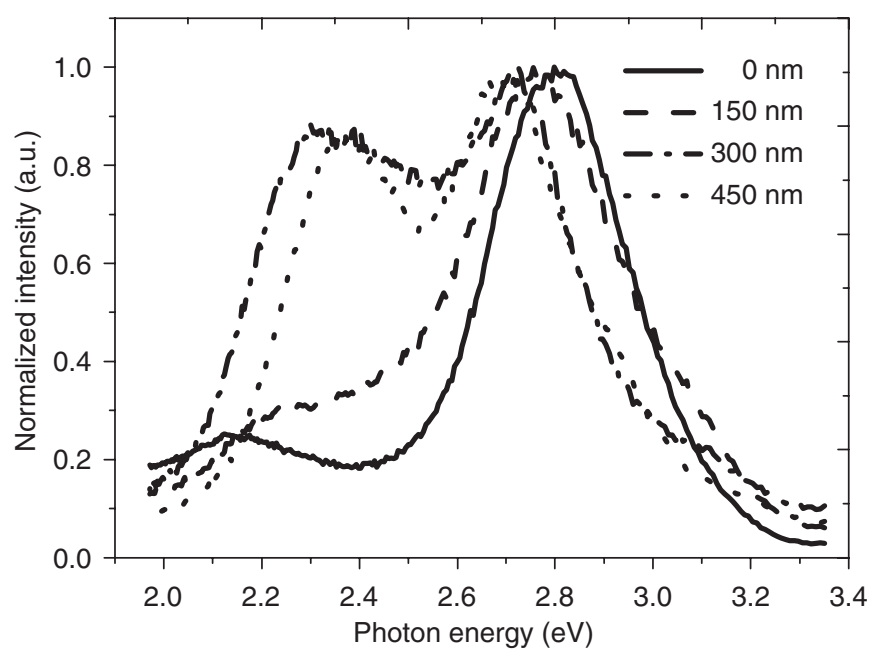

Fig. 6. Normalized CL spectra with electron energy of $2 \mathrm{keV}$ measured on the sample surfaces of different etching depths at $300 \mathrm{~K}$.

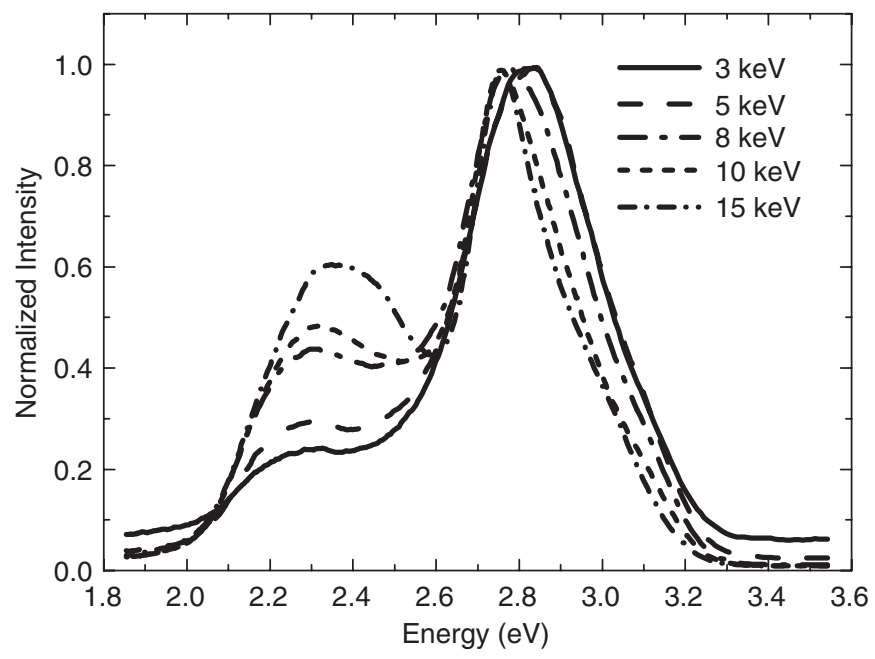

Fig. 7. Normalized CL spectra measured on the surface of the un-etched sample with various electron energies at $300 \mathrm{~K}$.

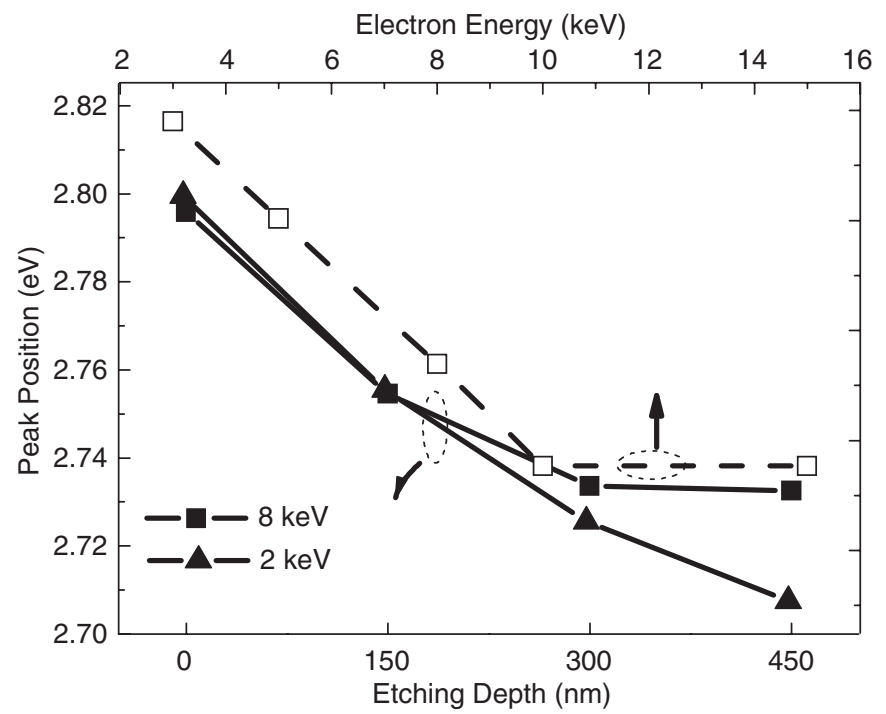

Fig. 8. CL spectral peak positions as functions of etching depth (lower coordinate) and electron energy (upper coordinate).

are about $88,210,450$, and $1300 \mathrm{~nm}$, respectively [8]. Therefore, in Fig. 8, the upper and lower coordinates of the same position do not represent the same effective probing depth. The saturation behavior in the two curves of square symbols is due to the complete coverage of the InGaN layer beyond $300 \mathrm{~nm}$ in depth. Such a saturation behavior is not observed in the measurement of shallow electron penetration on the samples of various etching depths. The $90 \mathrm{meV}$ red-shift in the curve of filled triangles shows the significant composition and/or nano-structure variations along the depth in the InGaN thin film sample.

\section{Discussions and conclusions}

Two possibilities can cause the observed red-shift trend with depth. First, the stronger clustering behavior in a deeper layer, as indicated in Fig. 3, may lead to lower potential minima such that effectively CL spectra are redshifted. Second, residual hetero-structure-induced strain may exist beyond the critical thickness. Such a strain field extends across a cluster, leading to the quantum-confined Stark effect (QCSE) and hence red shift of photon emission. The residual strain diminishes with growth thickness. Hence, CL spectral peak red shifts with depth. The red-shift trend also indicates that the indium-pulling effect is insignificant beyond the critical thickness in this sample. Different from the CL behaviors, the variation of PL spectral peak position with depth is weak and does not show a clear trend. This phenomenon can be attributed to the distribution of photo-generated carriers among different clusters of various potential minima. Hence, it is difficult to determine the ground energy level through the calibration of PL spectral peak. In this study, because the dry etching process may cause sample surface damage and $C L$ can reveal photon emission properties beneath surface (even with $2 \mathrm{keV}$ electron energy), the data of CL spectral 
peak position are more reliable in showing the depth dependent trend. Nevertheless, the difference between PL and EL measurements in such a clustering structure deserves further investigation.

In summary, we have studied the depth dependent variation of optical property beyond the critical thickness of an InGaN thin film. In the sample, both free-carrier and localized-state recombination activities could be observed. The emission peaks corresponding to the localized states in PL measurement became more prominent with increasing sample depth, implying stronger clustering in the deeper layers. Although the PL spectral peak varied weakly, that of CL, corresponding to the activities of the localized states, clearly red shifted with sample depth. The red shift trend was attributed to the stronger clustering behavior and possibly stronger QCSE (due to the residual strain beyond the critical thickness) in a deeper layer.

\section{Acknowledgement}

This research was supported by National Science Council, The Republic of China, under the grant of NSC 93-2210-M-002-006 and NSC 94-2215-E-002-015, and by US Air Force under the contracts AOARD-04-4026 and AOARD-05-4085.

\section{References}

[1] C.A. Parker, J.C. Roberts, S.M. Bedair, M.J. Reed, S.X. Liu, N.A. El-Masry, Appl. Phys. Lett. 75 (1999) 2776.
[2] M.J. Reed, N.A. El-Masry, C.A. Parker, J.C. Roberts, S.M. Bedair, Appl. Phys. Lett. 77 (2000) 4121.

[3] S. Pereira, M.R. Correia, E. Pereira, C. Trager-Cowan, F. Sweeney, K.P. O’Donnell, E. Alves, N. Franco, A.D. Sequeira, Appl. Phys. Lett. 81 (2002) 1287.

[4] Y.S. Lin, K.J. Ma, C. Hsu, S.W. Feng, Y.C. Cheng, C.C. Liao, C.C. Yang, C.C. Chou, C.M. Lee, J.I. Chyi, Appl. Phys. Lett. 77 (2000) 2988.

[5] S.W. Feng, Y.C. Cheng, Y.Y. Chung, C.C. Yang, M.H. Mao, Y.S Lin, K.J. Ma, J.I. Chyi, Appl. Phys. Lett. 80 (2002) 4375.

[6] S.W. Feng, Y.C. Cheng, Y.Y. Chung, C.C. Yang, K.J. Ma, C.C. Yan, C. Hsu, J.Y. Lin, H.X. Jiang, Appl. Phys. Lett. 82 (2003) 1377.

[7] Y.S. Lin, K.J. Ma, C. Hsu, Y.Y. Chung, C.W. Liu, S.W. Feng, Y.C. Cheng, C.C. Yang, M.H. Mao, H.W. Chuang, C.T. Kuo, J.S. Tsang, T.E. Weirich, Appl. Phys. Lett. 80 (2002) 2571.

[8] S.W. Feng, T.Y. Tang, Y.C. Lu, S.J. Liu, E.C. Lin, C.C. Yang, K.J. Ma, C.H. Shen, L.C. Chen, K.H. Kim, J.Y. Lin, H.X. Jiang, J. Appl. Phys. 95 (2004) 5388.

[9] K. Hiramatsu, Y. Kawaguchi, M. Shimizu, N. Sawaki, T. Zheleva, R.F. Davis, H. Tsuda, W. Taki, N. Juwano, K. Oki, MRS Internet J. Nitride Semicond. Res. 2 (1997) 6.

[10] S. Pereira, M.R. Correia, E. Pereira, K.P. O’Donnell, C. TragerCowan, F. Sweeney, E. Alves, Phys. Rev. B 64 (2001) 205311.

[11] M. Hao, H. Ishikawa, T. Egawa, C.L. Shao, T. Jimbo, Appl. Phys. Lett. 82 (2003) 4702.

[12] S.W. Feng, E.C. Lin, T.Y. Tang, Y.C. Cheng, H.C. Wang, C.C. Yang, K.J. Ma, C.H. Shen, L.C. Chen, K.H. Kim, J.Y. Lin, H.X. Jiang, Appl. Phys. Lett. 83 (2003) 3906.

[13] S.C. Bayliss, P. Demeester, I. Fletcher, R.W. Martin, P.G Middleton, I. Moerman, K.P. O'Donnell, A. Sapelkin, C. TragerCowan, W. Van Der Stricht, C. Young, Mat. Sci. Eng. B 59 (1999) 292.

[14] W. Van der Stricht, I. Moerman, P. Demeester, L. Condidine, E.J. Thrush, J.A. Crawley, MRS Internet J. Nitride Semicond. Res. 2 (1997) 16. 